\title{
Power-law/exponential transport of electromagnetic field in one-dimensional metallic nanoparticle arrays
}

\author{
Gang Song ${ }^{1} \&$ Wei Zhang ${ }^{1,2}$ \\ ${ }^{1}$ Institute of Applied Physics and Computational Mathematics, P. O. Box 8009(28), \\ Beijing 100088, P. R. China \\ 2 Beijing Computational Science Research Center, Beijing 100084, P. R. China \\ E-mail: zhang_wei@iapcm.ac.cn
}

\begin{abstract}
Based on the coupled-dipole analysis and finite-difference time-domain simulation, we have investigated the surface plasmon propagation in one-dimensional metallic nanoparticle (NP) chains. Our systematic studies reveal that the interplay between the localized plasmon excitation and the lattice collective behavior leads to two phases (I and II) of different electromagnetic (EM) field transport properties. In phase I, the EM field decays follow the power-law. In phase II, the EM field shows the exponential decay in the short distance regime and the power-law decay in the long distance regime. Moreover, universal power-law exponents have been found in the long propagation distance. The two different EM field propagation behaviors can be transformed to each other by tuning the parameters of the excitation fields and/or those of the NP chains. The EM field transport mechanisms we have found are very useful in the design of plasmonic waveguide with both strong field confinement and efficient field/energy transfer, which has important applications in integrated nanophotonic circuits.
\end{abstract}

Keywords: surface plasmons, propagations, power-law decay

\section{Introduction}

Extensive studies have been performed on the optical properties and their applications of metallic nanoparticles (NPs), especially the noble metal (such as the gold or the silver) NPs. Metallic NP arrays show interesting collective behaviors (in the

absorption/reflection/transmission etc.) [1, 2, 3, 4, 5, 6] due to the combination effect of near field and far field, for example, Wood anomaly in the reflection/absorption spectra. Metallic NPs arranged in a line can be used as a waveguide for localized surface plasmon (LSP) propagation [7, 8, 9, 10, 11, 12, 13, 14, 15, 16, 17, 18, 19, 20, 21, 22, 23, 24, 25, 26. 27]. The dispersion relationships of the modes for LSP propagating in the chain have been analyzed in both theory and experiment [9, 10, 11, 12, A series of theoretical methods such as the Mie scattering method were used to describe the characteristics of LSP propagation in metallic NP chains under specific conditions [23, 24, 25, 26, 27]. 
There are a lot of pervious works that point out the exponential decay as the main behavior of the energy transport in metallic nanoparticle chain in most conditions. Some calculation results showed that the non-exponential decay appeared in metallic NP chains [20, 23]. In experiment, the propagation behavior of LSPs with the bright mode showed the non-exponential decay in the chain with close-packed NPs 16. Though many theoretical and experimental studies have been performed in the past decades, the characteristics of LSP propagating in metallic NP arrays are still unclear. It is very important to clarify the propagation behavior of LSP in metallic NP chains, which is very useful in the area of optical communications.

In this paper, taking the silver NP arrays as the sample systems, we apply the coupled-dipole (CD) analysis and the finite-difference time-domain (FDTD) simulation to explore the characteristics of LSP propagating in metallic NP chains. The interplay between the localized plasmon excitation in the individual NP and the lattice collective behavior leads to two phases of different electromagnetic (EM) field transport properties. New propagation mechanisms/properties including power-law/exponential decay of EM fields and universal power-law exponents have been found.

\section{Two phases of EM field propagation}

In our system, a series of $\mathrm{Ag}$ NPs with the radius $R$ are arranged in a line with the lattice constant $l$ and the incident field polarization angle with respect to the direction of the chain is $\theta$ (see the schematic diagram in figure $1(\mathrm{a})$ ). In the coupled-dipole analysis, only the LSP on the first NP is excited by incident light, which could be realized by using a local tip to guide the light onto the first NP in experiment. We first consider the cases of the incident light polarization perpendicular to/parallel to the chain.

The transport of the EM field in one-dimensional (1D) NP chains is mainly determined by the competition between the loss and the interaction among the NPs, which is the combination effect of the individual NP plasmonic property and the collective behavior from the lattice. The plasmonic property of a single NP can be described by the polarizability $\alpha_{s}$ as [28]:

$$
\alpha_{s}=\frac{3 i}{2 k^{3}} \frac{\mu m^{2} j_{1}(m \rho)\left[\rho j_{1}(\rho)\right]^{\prime}-\mu_{1} j_{1}(\rho)\left[m \rho j_{1}(\rho)\right]^{\prime}}{\mu j_{1}(m \rho)\left[\rho h_{1}^{1}(\rho)\right]^{\prime}-\mu_{1} h_{1}^{1}(\rho)\left[m \rho j_{1}(\rho)\right]^{\prime}}
$$

where $\mu$ and $\mu_{1}$ are the magnetic permeabilities of the NP and the background, $k$ is the wave-vector in the vacuum, $m$ is the ratio of the index of the metal to that of the background, the dielectric constant of $\mathrm{Ag}$ is obtained from the reference [29], $j_{1}$ and $h_{1}^{1}$ are the spherical Bessel functions and $\rho=k R$. This expression is suitable to characterize the optical responses of sphere metal nanoparticles with the radius up to tens of nanometers [28, which also agree with the results calculated by the FDTD method. The collective behavior is due to the coupling between the NPs at different lattice positions. In the cases of $l \gg R$, the interaction between the NP at position $n l$ and the other NP at different lattice position $n^{\prime} l$ can be described as dipole coupling 
and can be written as [24]:

$$
G_{k}(r)=\left(\frac{k^{2}}{|r|}+\frac{i k}{|r|^{2}}-\frac{1}{|r|^{3}}\right) e^{i k|r|}
$$

with $r=n l-n^{\prime} l$ for the incident light polarization perpendicular to the chain and

$$
G_{k}(r)=\left(-\frac{2 i k}{|r|^{2}}+\frac{2}{|r|^{3}}\right) e^{i k|r|}
$$

for the incident light polarization along the chain. The coupled-dipole approach works well in the case of $l \geq 3 R[24,25,26,27,28$, which we mainly focus on. Here we apply it to illustrate the propagation characteristic of the silver NP chains. By solving the coupled-dipole equations for the dipole moments $D_{k}(n l)$ for NPs at position $n l$,

$$
D_{k}(n l)=\alpha_{s}\left[E_{n}+\Sigma_{n^{\prime}} G_{k}\left(n l-n^{\prime} l\right) D_{k}\left(n^{\prime} l\right)\right]
$$

with the incident field $E_{n}=E \delta_{n, 0}$, we obtain the dipole moment of each NP in the chain as [24]:

$$
D_{k}(n l)=E \int_{-\pi / l}^{\pi / l} \frac{\exp (i q n l)}{1 / \alpha_{s}-S(k, q)} \frac{l d q}{2 \pi},
$$

where $S(k, q)=2 \Sigma_{n>0} G_{k} \cos (q n l)$. The analytical structure of $1 / \alpha_{s}-S(k, q)$ has important impact on the propagation characteristics. The systems show quite different EM field transport behaviors depending on whether the resonant condition $\operatorname{Re}\left[1 / \alpha_{s}-\right.$ $S(k, q)]=0$ can be satisfied. The important role of the analytical structure of $1 / \alpha_{s}-S(k, q)$ reflects the interplay between the individual NP plasmonic properties $\left(\alpha_{s}\right)$ and the collective behavior $(S(k, q))$.

We firstly consider the cases with the incident light polarization perpendicular to the chain $\left(\theta=90^{\circ}\right)$. Here, we use the lattice constant $l=180 \mathrm{~nm}$. The chain with NPs of $R=50 \mathrm{~nm}$ is studied to demonstrate the EM field propagating in Ag NP chains for an incident wavelength $\lambda=320 \mathrm{~nm}$ as shown in Figure 1. Here $D_{k}$ is normalized as $D_{k} / E \alpha_{s}$. The linear relation of $\ln \left(\left|I_{D}\right|^{1 / 2}\right)$ [the local field intensity $I_{D} \propto\left|D_{k}\right|^{2}$ ] versus the logarithm of the propagation distance $(\ln (x))$ implies an power-law decay of the EM field for $\lambda=320 \mathrm{~nm}$.

At this wavelength, $\operatorname{Re}\left[1 / \alpha_{s}-S(k, q)\right]$ is always nonzero as shown in Figure 2, then the field decay is according to a power law. At the wavelength $\lambda=320 \mathrm{~nm}$, the absolute value of $1 / \alpha_{s}$ is larger than that of $S(k, q)$. From Equ.(5) we can use perturbation theory and obtain $D_{k}=\alpha_{s}^{2} S_{k} E=\left(\alpha_{s} E\right) S_{k} \alpha_{s}$, where $S_{k}(n l)=\int e^{i q n l} S(k, q) l d q / 2 \pi$. Here we have the physical picture: $\left(\alpha_{s} E\right)$ describes the dipole moment of a NP with polarizability $\left(\alpha_{s}\right), S_{k}$ describes the propagation of the dipole field, the second $\alpha_{s}$ describes the coupling with the other NP. $D_{k}$ or $S_{k}$ shows a universal power-law decay behavior, i.e. $D_{k} \sim 1 / x^{\beta}, \beta=1$ in the long propagation distance limit. We fit the data from Figure 1(b) and obtain the slope -1.07. The difference between the fitting power-law exponent 1.07 and $\beta=1$ is due to the finite fitting propagation distance.

The EM field propagation is determined by the analytical structure of $1 / \alpha_{s}-S(k, q)$ (as seen from Equ. (5)), which depends on the wavelength. Then, we take another 

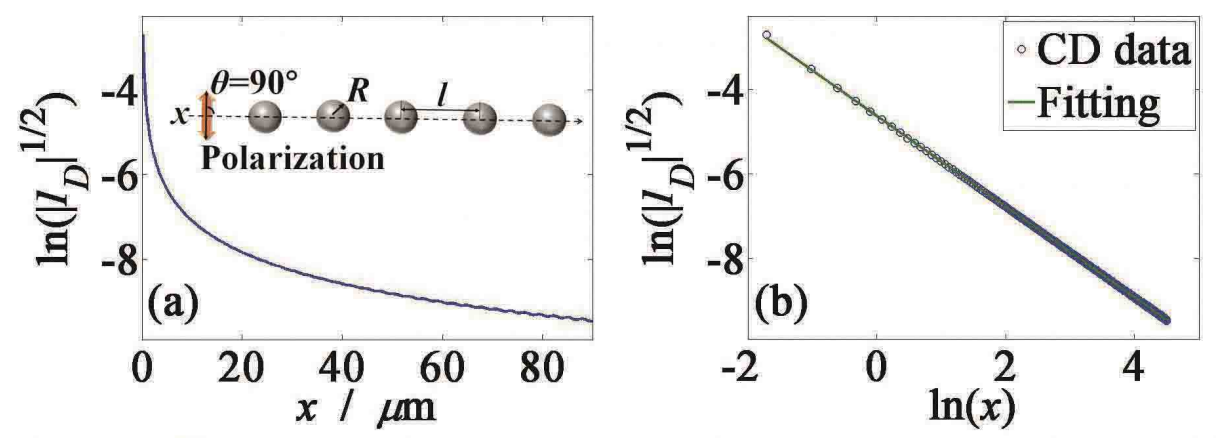

Figure 1. (Color online) $\ln \left(\left|I_{D}\right|^{1 / 2}\right)$ versus the propagation distance $x$ and $\ln (x)$ for $\lambda=320 \mathrm{~nm}$, respectively. The incident light polarization is perpendicular to the chain $\left(\theta=90^{\circ}\right)$. Here CD data is the computation results based on coupled-dipole method.

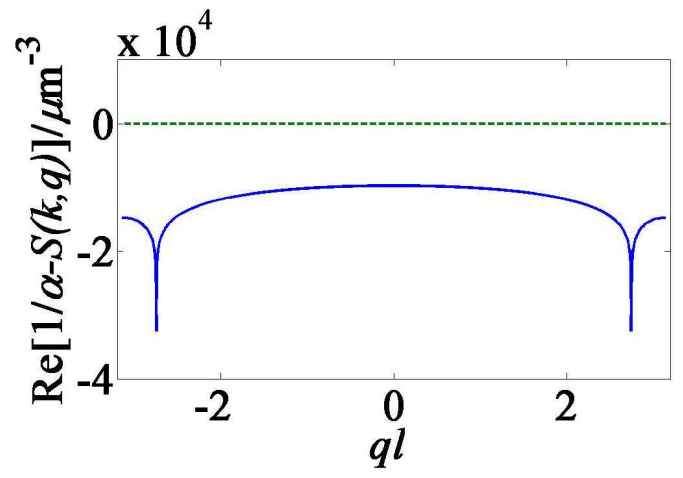

Figure 2. (Color online) $R e\left[1 / \alpha_{s}-S(k, q)\right]$ versus $q l$ for $\lambda=320 \mathrm{~nm}$. The incident light polarization is perpendicular to the chain.

wavelength to show the other phase of the EM field propagation. $\lambda=440 \mathrm{~nm}$ is adopted as the working wavelength. $\ln \left(\left|I_{D}\right|^{1 / 2}\right)$ versus the propagation distance $x$ and $\ln (x)$ are shown in Figure 3, respectively. At $\lambda=440 \mathrm{~nm}$, the linear relation of $\ln \left(\left|I_{D}\right|^{1 / 2}\right)$ versus $x$ indicates the exponential decay in the regime of $x$ from the beginning to about $4 \mu m$ as shown in the insert of Figure $3(\mathrm{a})$, while the linear relation of $\ln \left(\left|I_{D}\right|^{1 / 2}\right)$ versus $\ln (x)$ implies the power-law decay in the regime of $\ln (x)$ from $1.81(x \sim 6.12 \mu m)$ to the end. Thus, the EM field propagates following an exponential law in the short distance regime and a power-law in the long distance regime (exponential decay + power-law decay).

The curve of $\operatorname{Re}\left[1 / \alpha_{s}-S(k, q)\right]$ versus $q l$ for $\lambda=440 \mathrm{~nm}$ is shown in Figure 4 . One can see that there are solutions $q_{j}(j=1,2 \ldots)$ to the equation $\operatorname{Re}\left[1 / \alpha_{s}-S(k, q)\right]=0$. As seen from Figure 4 , the solution $q_{j}$ is around $\pm k$. It is related to the singularity around $q \pm k$, which is caused by the long-range coulomb interaction and has the same origin as the Wood anomaly. For simplicity, we consider a solution $\bar{q}$. The generalization to the case with multiple solutions is straightforward. Near $\bar{q}, 1 / \alpha_{s}-S(k, q) \simeq$ $\left.\frac{\partial \operatorname{Re} S(k, q)}{\partial q}\right|_{q=\bar{q}}(q-\bar{q})+i \eta \equiv Y, \eta=\operatorname{Im}(1 / \alpha-S(k, \bar{q}))$. Then, we can rewrite Equ.(5) into 

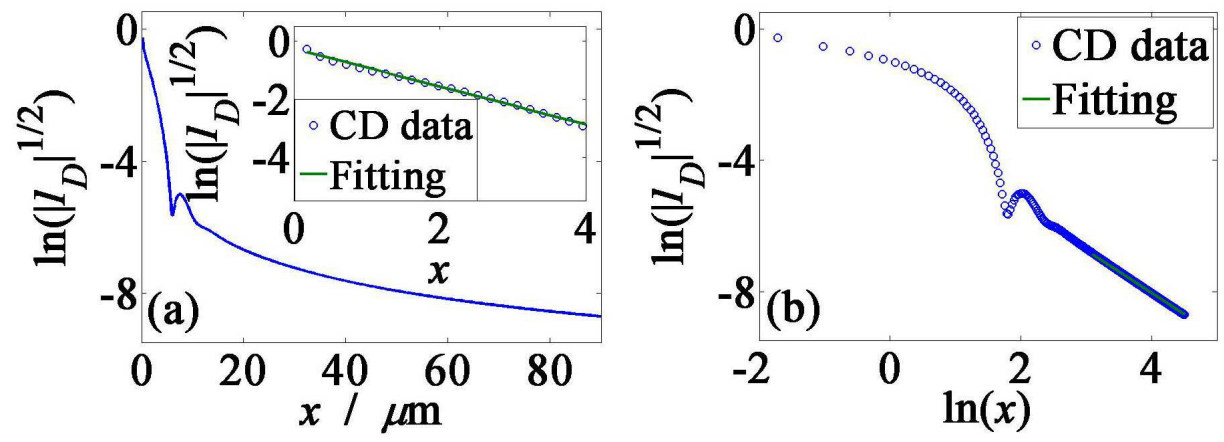

Figure 3. (Color online) $\ln \left(\left|I_{D}\right|^{1 / 2}\right)$ versus the propagation distance $x$ and $\ln (x)$ for $\lambda=440 \mathrm{~nm}$, respectively. The incident light polarization is perpendicular to the chain.

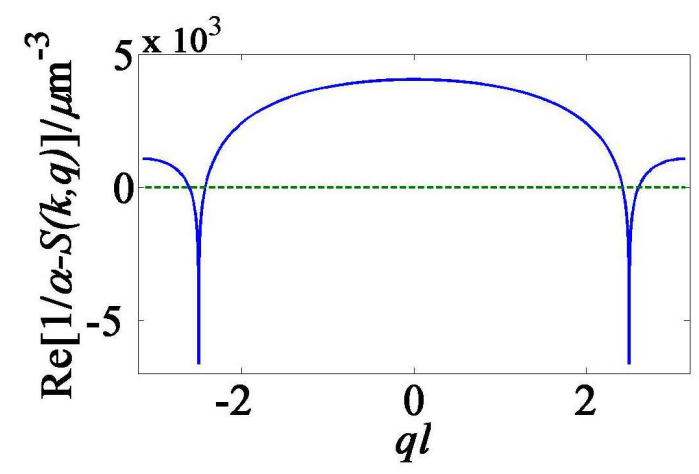

Figure 4. (Color online) $R e\left[1 / \alpha_{s}-S(k, q)\right]$ versus $q l$ for $\lambda=440 \mathrm{~nm}$. The incident light polarization is perpendicular to the chain.

two parts as:

$$
D_{k}(n l)=E \int_{-\pi / l}^{\pi / l} \frac{e^{i q n l}}{Z} \frac{l d q}{2 \pi}=E \int_{-\pi / l}^{\pi / l} \frac{e^{i q n l}}{Y} \frac{l d q}{2 \pi}+E \int_{-\pi / l}^{\pi / l} \frac{e^{i q n l}}{X} \frac{l d q}{2 \pi},
$$

where $Z=1 / \alpha_{s}-S(k, q), 1 / X=1 / Z-1 / Y$. The first term on the right hand of Equ. (6) is in the form of $e^{-n l / L}\left(L=\frac{1}{\eta}\left|\frac{\partial S(k, q)}{\partial q}\right|_{q=\bar{q}}\right)$, showing an exponential decay behavior. While for the second term, $\left.\operatorname{Re}(X) \simeq \frac{2(\partial S / \partial q)^{2}}{\partial^{2} S / \partial q^{2}}\right|_{q=\bar{q}}$ at $q=\bar{q}$. Considering the very fast change of $Z$ with $q$ near $\bar{q}$ and the regularization of the singularity of $1 / Z$ $(1 / Z \rightarrow 1 / X=1 / Z-1 / Y)$, the second term shows power-law decay as that in Figure 1. We can obtain the power-law exponent as 1.29 from fitting the data in Figure 3(b).

Then, the polarization of incident light along the chain $\left(\theta=0^{\circ}\right)$ is considered. By observing Equ. (2) and Equ.(3), we can see that the Wood anomaly-like singularity disappears and expect some changes in the propagation/transport behavior. The curves of $\ln \left(\left|I_{D}\right|^{1 / 2}\right)$ versus the propagation distance $x$ and $\ln (x)$ for $R=50 \mathrm{~nm}, l=180 \mathrm{~nm}$, $\lambda=320 \mathrm{~nm}$ and $440 \mathrm{~nm}$ are shown in Figure 5, respectively. The clear linear relation of $\ln \left(\left|I_{D}\right|^{1 / 2}\right)$ versus $\ln (x)$ indicates a power-law decay of the EM fields.

As shown in Figure 6, there is no solution to the equation $\operatorname{Re}\left[1 / \alpha_{s}-S(k, q)\right]=0$ for these two wavelengths $\lambda=320 \mathrm{~nm}$ and $440 \mathrm{~nm}$. It is quite different from the case with the polarization perpendicular to the chain (Figure 2 and Figure 4), which is related to the 

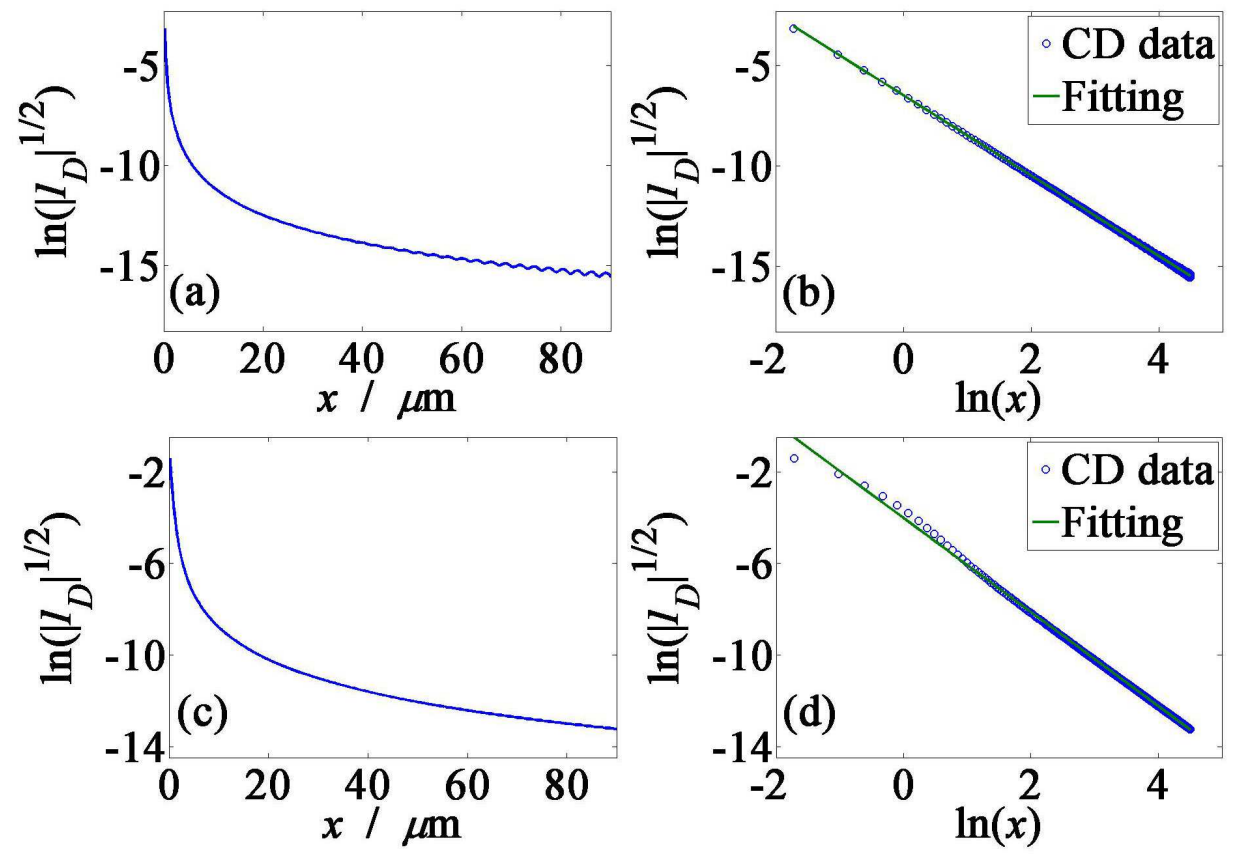

Figure 5. (Color online) $\ln \left(\left|I_{D}\right|^{1 / 2}\right)$ versus the propagation distance $x$ and $\ln (x)$ for $\lambda=320 \mathrm{~nm}(\mathrm{a}-\mathrm{b})$ and $440 \mathrm{~nm}(\mathrm{c}-\mathrm{d})$, respectively. The incident light polarization is parallel to the chain.
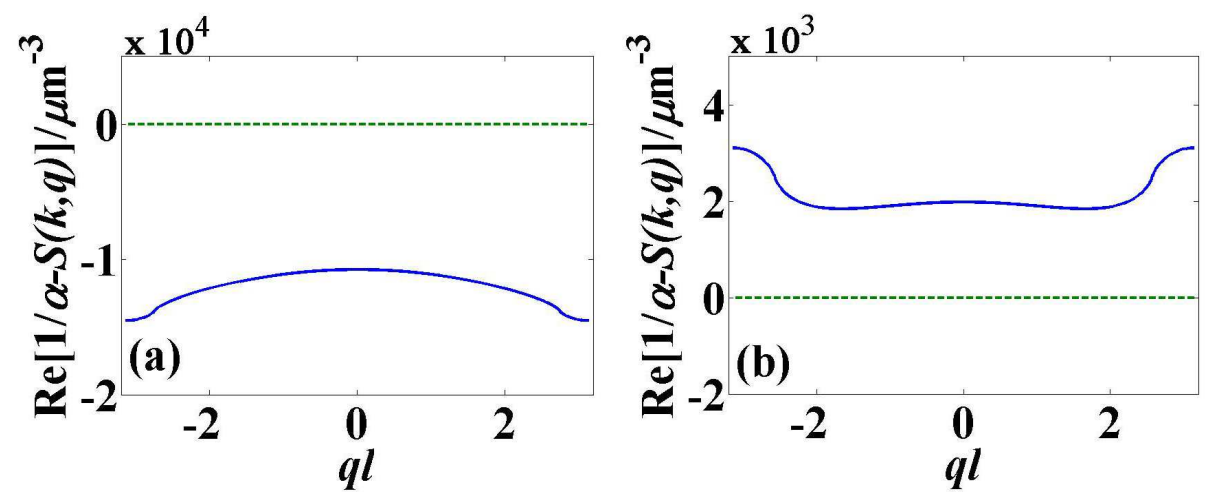

Figure 6. (Color online) $\operatorname{Re}\left[1 / \alpha_{s}-S(k, q)\right]$ versus $q l$ for $\lambda=320 \mathrm{~nm}$ (a) and $440 \mathrm{~nm}$ (b), respectively. The incident light polarization is parallel to the chain.

disappearance of Wood anomaly-like singularity. Moreover the power index should also be different. From Equ.(3), the universal power-law exponent should be 2 in this case. We fit the data from Figure 5(b) and 5(d) and obtain the slope -2.01 and -2.05 in the long propagation distance regime, respectively. There may also exist a small intermediate wavelength range in which there are some $q_{0}$ to make $\operatorname{Re}\left[1 / \alpha_{s}-S(k, q)\right]=0$. And the propagation behavior is the exponential decay + power-law decay.

Here we would like to note that when there is zero of $\operatorname{Re}\left[1 / \alpha_{s}-S(k, q)\right]$, the system shows a combination of exponential decay and power-law decay. In the long distance regime, the power-law decay dominates. In the short distance regime, it is also possible 


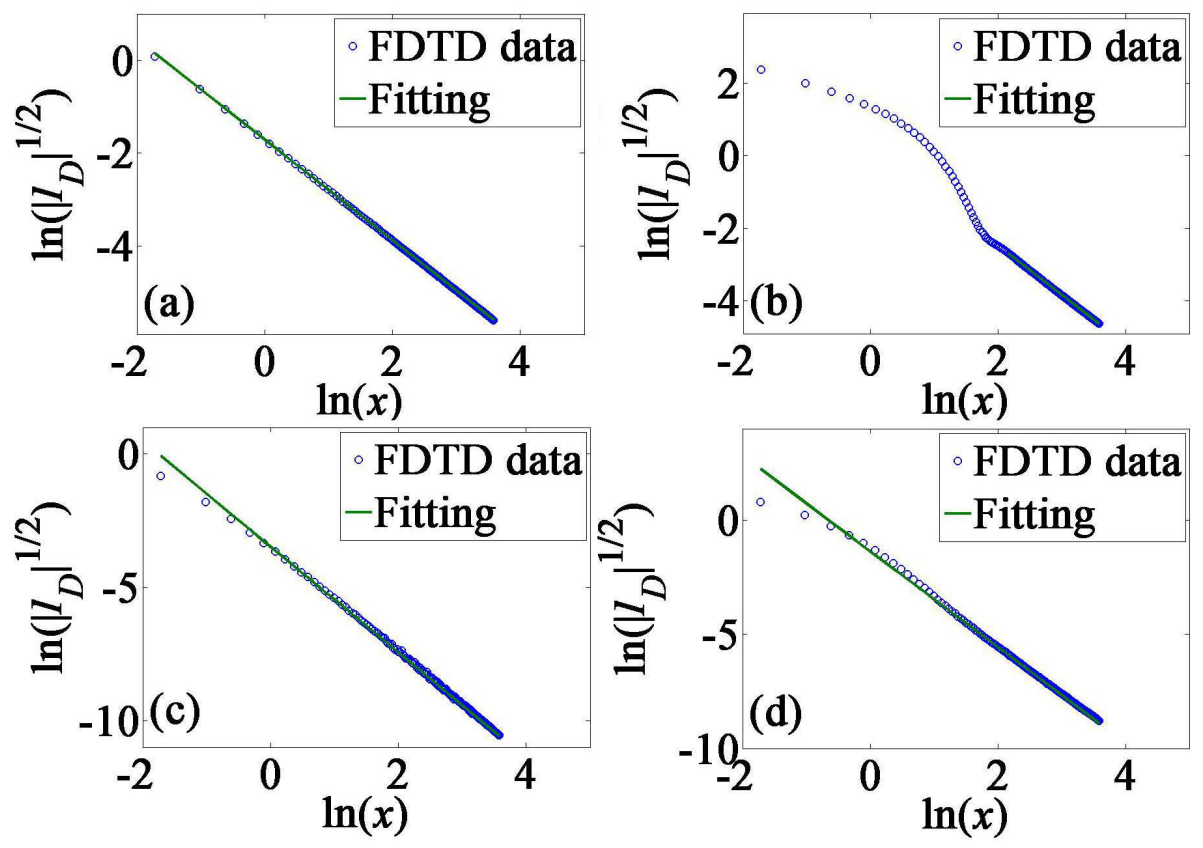

Figure 7. (Color online) $\ln \left(\left|I_{D}\right|^{1 / 2}\right)$ versus $\ln (x)$ for the polarization of the source dipole perpendicular to $(\mathrm{a}, \mathrm{b}) /$ parallel to $(\mathrm{c}, \mathrm{d})$ the chain with $\lambda=320 \mathrm{~nm}(\mathrm{a}, \mathrm{c})$ and $440 \mathrm{~nm}(\mathrm{~b}, \mathrm{~d})$, respectively. $I_{D}$ is the EM field intensity, and $x$ is the propagation distance.

that the contribution of the power-law decay term is larger than that of the exponential decay term. Then the system shows the effective power-law decay behavior, for example for the case with $\lambda=900 \mathrm{~nm}$.

To further support our study based on coupled-dipole analysis, we perform the FDTD simulation of the EM field propagation. In the simulation, the chain has 200 silver nanoparticles with radius of $50 \mathrm{~nm}$ and the lattice constant is $180 \mathrm{~nm}$. A dipole source is located at a distance of $l=180 \mathrm{~nm}$ to the first nanoparticle along the chain. The polarization of the dipole source is perpendicular/parallel to the chain. The detecting point is on the side of the nanoparticle with $2.5 \mathrm{~nm}$ away from the interface of the nanoparticle along the direction of the dipole. The incident wavelengths are $320 \mathrm{~nm}$ and $440 \mathrm{~nm}$. The gird is $2.5 \mathrm{~nm}$. The detecting EM field intensity is $I_{D} \cdot \ln \left(\left|I_{D}\right|^{1 / 2}\right)$ versus $\ln (x)$ ( $x$ the propagation distance) is shown in Figure 7.

From Figure 7(a) and 7(b), we find two different field transport characteristics for the source dipole perpendicular to the chain. The linear relation of $\ln \left(\left|I_{D}\right|^{1 / 2}\right)$ versus $\ln (x)$ in Figure $7(\mathrm{a})$ indicates a power-law decay of EM field with the slope of -1.08 for $\lambda=320 \mathrm{~nm}$. Figure 3 shows an exponential decay+power law decay of the EM field for $\lambda=440 \mathrm{~nm}$ based on CD method. Here, as shown in Figure $7(\mathrm{~b})$, the linear relation of $\ln \left(\left|I_{D}\right|^{1 / 2}\right)$ versus $\ln (x)$ is in the regime from about $1.81(x \sim 6.12 \mu m)$ to the end, which implies an exponential decay + power-law decay of EM field with the slope of - 1.35 for $\lambda=440 \mathrm{~nm}$. The derivation of the exponent from 1 is due to the finite fitting regime. From Figure $7(\mathrm{c})$ and $7(\mathrm{~d})$, the linear relations of $\ln \left(\left|I_{D}\right|^{1 / 2}\right)$ versus $\ln (x)$ for the the 
Table 1. The power-law exponents from the couple dipole (CD) calculations and FDTD simulations.

\begin{tabular}{lll}
\hline & CD & FDTD \\
\hline$\lambda=320 \mathrm{~nm}, \perp$ & -1.07 & -1.08 \\
$\lambda=440 \mathrm{~nm}, \perp$ & -1.29 & -1.35 \\
$\lambda=320 \mathrm{~nm}, \|$ & -2.01 & -1.99 \\
$\lambda=440 \mathrm{~nm}, \|$ & -2.05 & -2.09 \\
\hline
\end{tabular}

polarization of the source dipole along the chain indicate the power-law decay of EM field with the slopes of -1.99 and -2.09 , respectively. The power-law exponents based on the couple dipole calculations and the FDTD simulations are listed in Table 1. As shown in Table 1, EM field transport characteristics and the power-law exponents from the FDTD simulations agree well with those based on coupled-dipole analysis.

\section{Universality and modulation of EM field propagation}

We have shown that there are two phases of the EM field propagation in 1D metallic NP chains, which are determined by the analytical structure of $1 / \alpha_{s}-S(k, q)$. The parameters of the excitation field and the structure of the NP chains have important impacts on the EM field transport behavior. As discussed above, the two phases of the EM field propagation can be transformed to each other by tuning the wavelength of the excitation field.

We further explore the EM field propagation with excitation fields of different polarizations. The calculation results in Figure 8 show that the EM field propagates according to power-law for $\lambda=320 \mathrm{~nm}$. The power-law exponents is 1.08 for incident field polarization angles $30^{\circ}, 45^{\circ}, 60^{\circ}, 90^{\circ}$ with respect to the direction of the chain. The power-law exponents is 1.99 for the polarization angle $0^{\circ}$. In the presence of excitation fields with wavelength $\lambda=440 \mathrm{~nm}$ and polarization angles $30^{\circ}, 45^{\circ}, 60^{\circ}, 90^{\circ}$, the EM field propagates following exponential law in the short distance regime and power-law in the long-distance regime. The power-law exponents are 1.43, 1.41, 1.40, 1.35 for polarization angles $30^{\circ}, 45^{\circ}, 60^{\circ}, 90^{\circ}$. These power-law exponents obtained by fitting the $\ln \left(\left|I_{D}\right|^{1 / 2}\right)-\ln (x)$ curves in the regimes of finite propagation distance show the combination power-law decay of EM field with exponents 1 and 2. In the very long propagation distance regime, the leading contribution is the power-law decay with exponent 1 . For the case of incident field polarization angle $0^{\circ}$, the EM field propagates following the power law with exponent 2.09 (by fitting the calculation data in finite propagation distance).

The geometric parameters of the NP chain also play an important role in the EM field transport. The calculation results of EM field propagation in metallic chains with Ag NPs of radius $R=40 \mathrm{~nm}, 45 \mathrm{~nm}, 55 \mathrm{~nm}$ are shown in Figure 9. The general power-law decay of EM field has been found for the cases with the wavelength $\lambda=320 \mathrm{~nm}$ (incident 

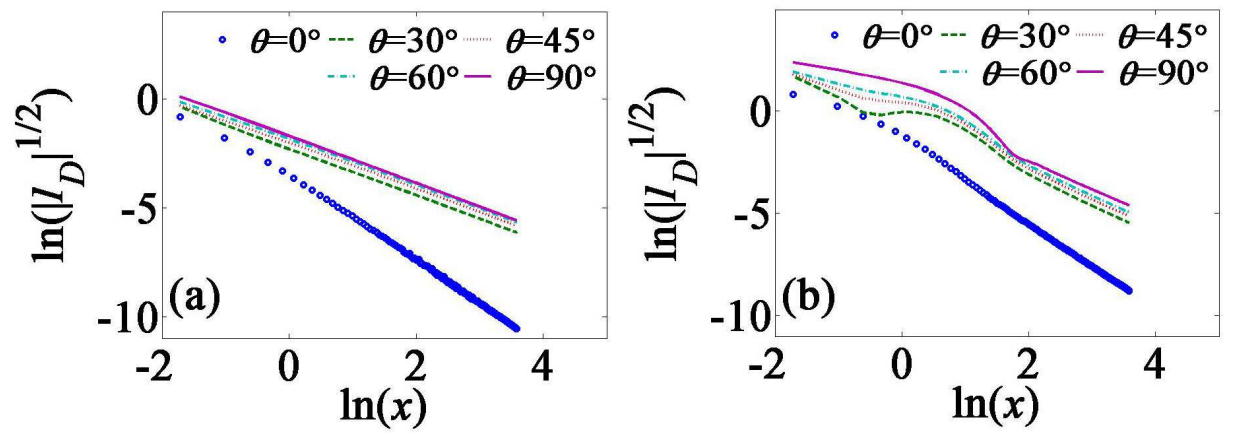

Figure 8. ((Color online) $\ln \left(\left|I_{D}\right|^{1 / 2}\right)$ versus $\ln (x)$ for the polarization angles $0^{\circ}, 30^{\circ}$, $45^{\circ}, 60^{\circ}, 90^{\circ}$ of the source dipole with respect to the direction of the Ag chain. (a) $\lambda=320 \mathrm{~nm}$ and (b) $\lambda=440 \mathrm{~nm}$.
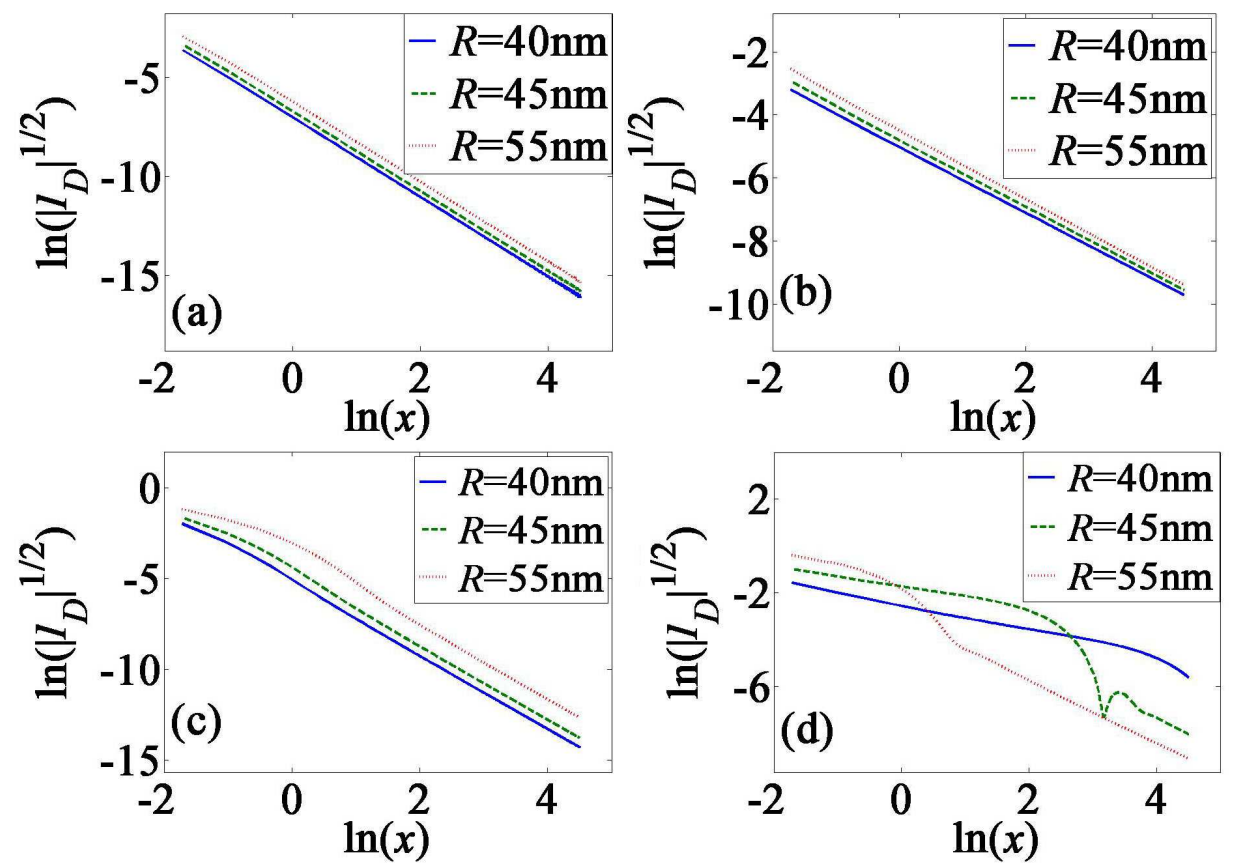

Figure 9. (Color online) $\ln \left(\left|I_{D}\right|^{1 / 2}\right)$ versus $\ln (x)$ for $\mathrm{Ag}$ chains with particles of radius $R=40 \mathrm{~nm}, 45 \mathrm{~nm}, 55 \mathrm{~nm}$, respectively. The polarization of the source dipole is parallel to $(\mathrm{a}, \mathrm{c}) /$ perpendicular to $(\mathrm{b}, \mathrm{d})$ the chain with $\lambda=320 \mathrm{~nm}(\mathrm{a}, \mathrm{b})$ and $440 \mathrm{~nm}(\mathrm{c}, \mathrm{d})$, respectively.

field polarization parallel to/ perpendicular to the chain) and the wavelength $\lambda=440 \mathrm{~nm}$ (the incident field polarization parallel to the chain) as shown in Figure 9(a), 9(b) and 9 (c). While for $\lambda=440 \mathrm{~nm}$ (incident field polarization perpendicular to the chain), the EM field transport behavior is exponential decay + power-law decay. For NPs with different sizes, the contributions from the exponential decay and the power-law decay are different, which leads to the change of the transition point (from the exponential decay to the power-law decay).

We have focused our studies on the systems with large lattice constant $(l>3 R)$, in which the CD method works well as supported by the FDTD simulations. By 

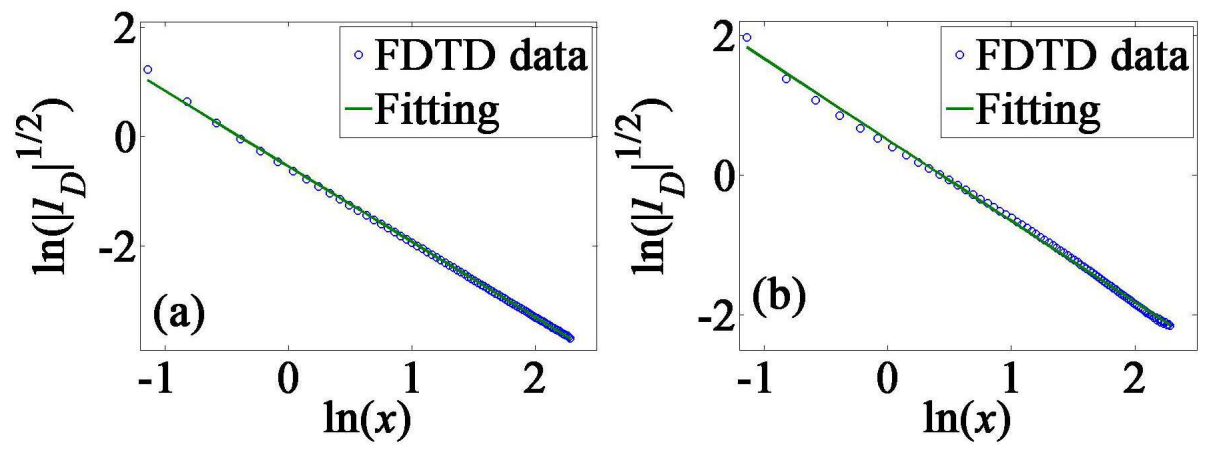

Figure 10. (Color online) $\ln \left(\left|I_{D}\right|^{1 / 2}\right)$ versus $\ln (x)$ for the polarization of the source dipole perpendicular to $\mathrm{Ag}$ chain for $\lambda=300 \mathrm{~nm}$ (a) and Au chain for $\lambda=514 \mathrm{~nm}$ (b), respectively. $I_{D}$ is the EM field intensity, and $x$ is the propagation distance.
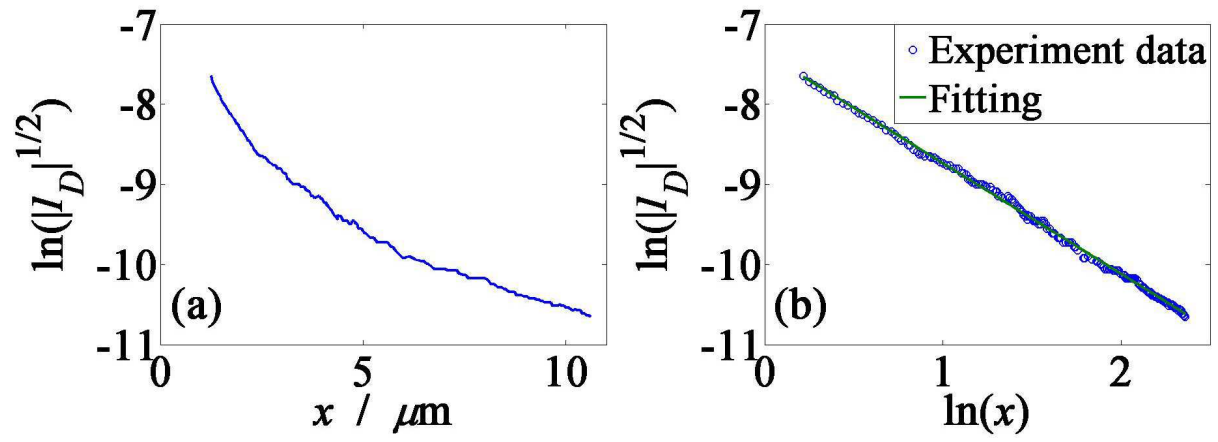

Figure 11. (Color online) (a) $\ln \left(\left|I_{D}\right|^{1 / 2}\right)$ versus the propogation distance $x$ based on the the experiment data at $\lambda=514 \mathrm{~nm}[16]$; (b) $\ln \left(\left|I_{D}\right|^{1 / 2}\right)$ versus $\ln (x)$ for $\lambda=514 \mathrm{~nm}$.

using CD method, we are able to provide analytical solutions and clear physical picture/mechanism, also gives the universal power-law exponents. We then consider the cases of lattice constant smaller than $3 R$. We use FDTD method to study the propagation behavior of NP chains with $l<3 R$. In the calculation we use the NP chain with $R=50 \mathrm{~nm}, l=120 \mathrm{~nm}$. Silver and Gold NPs are all considered to demonstrate the universality of the propagation property. The dielectric constants are obtained from the reference [29]. The incident field polarization is perpendicular to the chain and the wavelength $\lambda$ is $300 \mathrm{~nm}$ for Ag NP chain and $514 \mathrm{~nm}$ for Au NP chain. $\ln \left(\left|I_{D}\right|^{1 / 2}\right)$ versus $\ln (x)$ are shown in Figure 10. As shown in Figure 10, both the two lines show the linear relation with $\ln (x)$. We fit the two lines and the two slopes are -1.38 and -1.16 . These results point that the power-law decay may also appear in different metallic NP chains with different lattice constants.

The non-exponential decay is also pointed out in the experiment with close-packed NP chain [16]. The experiment data can be replotted in the double logarithm form as shown in Figure 11. The linear relation between $\ln \left(\left|I_{D}\right|^{1 / 2}\right)$ versus $\ln (x)$ ( $x$ the propagation distance) indicate a power-law decay of EM field. The pow-law exponent is 1.38. Hence, our analytical and simulation methods provide new ways to clarify the propagation behaviors in 1D metallic NP chains in previous studies [16, 20, 23]. 


\section{Conclusions}

In summary, we have studied the characteristics of the LSP propagation in onedimensional metallic nanoparticle chain based on the coupled-dipole calculations and FDTD simulations. The LSP propagation shows different behaviors in different phases, depending on the analytical structure of $1 / \alpha_{s}-S(k, q)=0$, which is determined by both the individual NP plasmonic property and the lattice collective effect. In phase I, a power-law decay of EM field has been identified. While in phase II, EM field propagates in the form of the exponential decay+power law decay, i.e., exponential decay in the short distance regime and power-law decay in the long distance regime. The universal power-law exponents have been found for EM field propagation in the long propagation distance regime. The results of FDTD simulations agree well with our theoretical predictions based on coupled-dipole method. It is found that the incident field (wavelength and polarization) and the geometric parameters of the NP chains have important impacts on the EM field transport properties. Our studies reveal a general picture of the EM field transport in nanoparticle chains, which have applications in the plasmonic waveguide and integrated nanophotonic circuits.

\section{Acknowledgement}

This work was partially supported by National Key Research and Development Program of China (Grant No. 2017YFA0303400), National Natural Science Foundation of China (Grant Nos. 11774036, 11374039).

\section{References}

[1] Wood R W 1902 Proc. Phys. Soc. London 18 269-275.

[2] Auguié B and Barnes W L 2008 Phys. Rev. Lett. 101143902.

[3] Haes A J and Van Duyne R P 2004 Anal Bioanal Chem. 379 920-930.

[4] Fischer M P, Schmidt C, Sakat E, Stock J, Samarelli A, Frigerio J, Ortolani M, Paul D J, Isella G, Leitenstorfer A, Biagioni P, and Brida D 2016 Phys. Rev. Lett. 117047401.

[5] Ma J and Pesin D A 2017 Phys. Rev. Lett. 118107401.

[6] Ling C W, Xiao M, Chan C T, Yu S F, and Fung K H 2015 Optics Express 232021.

[7] Maier S A, Kik P G, Atwater H A, Meltzer S, Harel E, Koel B E, Requicha A A G 2003 Nat. materials 2 229-232.

[8] Maier S A, Kik P G, and Atwater H A 2002 Appl. Phys. Lett. 81 1714-1716.

[9] Pike N A, and Stroud D 2013 J. Opt. Soc. Am. B 30 1127-1134.

[10] Park S Y and Stroud D 2004 Phys. Rev. B 69125418.

[11] Crozier K B, Togan E, Simsek E, and Yang T 2007 Opt. Express 15 17482-17493.

[12] Yang T, and Crozier K B 2008 Opt. Express 16 8570-8580.

[13] Kravets V V, Ocola L E, Khalavka Y, and Pinchuk A O 2015 Appl. Phys. Lett. 106053104.

[14] Stepanov A L, Krenn J R, Ditlbacher H, Hohenau A, Drezet A, Steinberger B, Leitner A, and Aussenegg F R 2005 Opt. Lett. 30 1524-1526.

[15] Xiao J J, Yakubo K, and Yu K W 2006 Appl. Phys. Lett. 89221503.

[16] SolisJr D, Willingham B, Nauert S L, Slaughter L S, Olson J, Swanglap P, Paul A, Chang W S, and Link S 2012 Nano Lett. 12 1349-1353. 
[17] SolisJr D, Paul A, Olson J, Slaughter L S, Swanglap P, Chang W S, and Link S 2013 Nano Lett. 13 4779-4784.

[18] Yin L L, Vlasko-Vlasov V K, Pearson J, Hiller J M, Hua J, Welp U, Brown D E, and Kimball C W 2005 Nano Lett. 5 1399-1402.

[19] Radko I P, Bozhevolnyi S I, Evlyukhin A B, Boltasseva A 2007 Opt. Express 15 6576-6582.

[20] Willingham B, and Link S 2011 Opt. Express 19 6450-6461.

[21] Barrow S J, Funston A M, Gomez D E, Davis T J, and Mulvaney P 2011 Nano Lett. 11 4180-4187.

[22] Février M, Gogol P, Aassime A, Megy R, Delacour C, Chelnokov A, Apuzzo A, Blaize S, Lourtioz J M, and Dagens B 2012 Nano Lett. 12 1032-1037.

[23] Quinten M, Leitner A, Krenn J R, and Aussenegg F R 1998 Opt. Lett. 23 1331-1333.

[24] Markel V A, and Sarychev A K 2007 Phys. Rev. B 75085426.

[25] Rasskazov I L, Karpov S V, and Markel V A 2013 Opt. Lett. 38 4743-4746.

[26] Rasskazov I L, Karpov S V, and Markel V A 2014 J. Opt. Soc. Am. B 31 2981-2989.

[27] Rasskazov I L, Karpov S V, and Markel V A 2014 Phys. Rev. B 90075405.

[28] Zou S L, Schatz G C 2006 Nanotechnology 17 2813-2820.

[29] Palik E 1985 Handbook of Optical Constant of Solids (Academic: San Diego) p350. 